\title{
THE ALCHEMY OF CHAOS: A SOUND ART SONIFICATION OF A YEAR OF TOURETTE'S EPISODES
}

\section{Thomas J. Mitchell}

\author{
Creative Technologies Lab \\ UWE, Bristol, UK \\ tom.mitchell@uwe.ac.uk
}

\author{
Jess Thom, Matthew Pountney \\ Touretteshero \\ London, UK \\ infodtouretteshero.com
}

\author{
Joseph Hyde \\ Bath Spa Univeristy \\ Bath, UK \\ j.hydeebathspa.ac.uk
}

\begin{abstract}
Touretteshero is the name of a organisation that aims to raise awareness of Touette's syndrome by sharing and celebrating the creativity and humour of the involuntary vocal and movement tics that characterise the condition. This paper documents the development of a Touretteshero project called The Alchemy of Chaos, a sound art piece that translates a year of intensive ticcing episodes (or 'ticcing fits') into a six minute sonification. The work emphasises both the faithful representation of data and the aesthetic sound quality, drawing techniques and ideas from sound design for film, which is often used to convey information about a visual scene in ways that can be used for sonfication. Specifically, the work uses Chion's elements of auditory setting: short punctual sounds that can express locations with minimal sonic references. Sound parameters are also classified into groups that have 'data significance' and those that do not, with aesthetic interventions limited to those parameters that do not impact on data transparency. The resulting piece was included within a keynote talk at the Royal Albert Hall in the UK and the paper includes a qualitative reflection on the work and the potential value that sound design techniques for film can bring to the auditory display community.
\end{abstract}

\section{INTRODUCTION}

Gilles de la Tourette syndrome (Tourette's) is a neurological condition that is characterised by involuntary and uncontrollable movements (motor tics) and vocalisations (vocal tics). Estimates vary, but studies have shown that Tourette's affects between $0.4 \%$ and $5.0 \%$ of children [1]. The tics associated with Tourette's can vary in severity, from subtle muscle contractions to sustained and intense spasms that can appear like seizures [2].

This paper documents the sonification of a year of intense ticcing episodes recorded by an individual with Tourette's between 2011 and 2012. The records were kept initially to enable longitudinal analysis by medical practitioners but in this work the data are used to define the structure of a sound art piece, to reveal and share the human experiences of Tourette's. The paper begins with an introduction to the Touretteshero project before introducing the salient literature relevant to sonification and sound art. The methods and processes adopted for this work are presented and the final piece is then discussed. The paper concludes with remarks on the

(c) (1) (8) This work is licensed under Creative Commons Attribution Non Commercial 4.0 International License. The full terms of the License are available at http://creativecommons.org/licenses/by-nc/4.0 importance of aesthetic considerations in sonification design and, in particular, how sound design techniques from film sound have the capacity to enhance and humanise information when expressed as sound.

\section{TOURETTESHERO}

Individuals with Tourette's can often experience discriminatory behaviour in public spaces and often withdraw from social activities to avoid confrontation [3]. This lack of public understanding and the resulting social isolation can have a negative impact on the lived expedience and quality of life of people with Tourette's. 'Touretteshero' is both the alias of Jess Thom and the name of an organisation that was set up in 2010 to raise awareness of Tourette's and its challenges.

Jess was diagnosed with Tourette's in her early twenties and at the time of writing, her tics are frequent and varied. Her vocal tics produce combinations of sounds and words with the occasional appearance of offensive language, referred to as Coprolalia, which affects $10 \%-15 \%$ of individuals with Tourette's [4]. However, more frequently Jess's vocalisations produce highly creative and humorous phrases that originally inspired Touretteshero. Jess's movement tics include, blinking, shrugging, jumping, head jerking and leg bending, which when combined make walking difficult, so Jess uses a wheelchair. Sometimes, these movements intensify into 'ticcing fits': distinct periods of overpowering and constant motor movements that typically last between 10 minutes and an hour (although sometimes considerably longer).

A hallmark of Touretteshero is the celebration of the humour and creativity of Jess's tics, embracing the utterances and movements as a source of inspiration for a range of artistic outputs. These outputs typically involve collaborations with artists to create imagery inspired by Jess's vocal tics, see Figure 1(a) and (b), the publication of biographical writings [5] and events that include performers with Tourette's, Figure 1(c). This theme of creativity motivated this sonification work: the desire to translate a medical record of ticcing fits, that represent an immense amount of discomfort into something engaging and beautiful.

\section{SONIFICATION, SOUND DESIGN AND MUSIC}

The relationship between sonification and music has formed the basis of much discussion and disagreement in the auditory display community. For example, Vickers and Hogg go some way to reduce the differences between sonification and music to the perceptions of the listener [6,7], Scaletti argues that both endeavours 


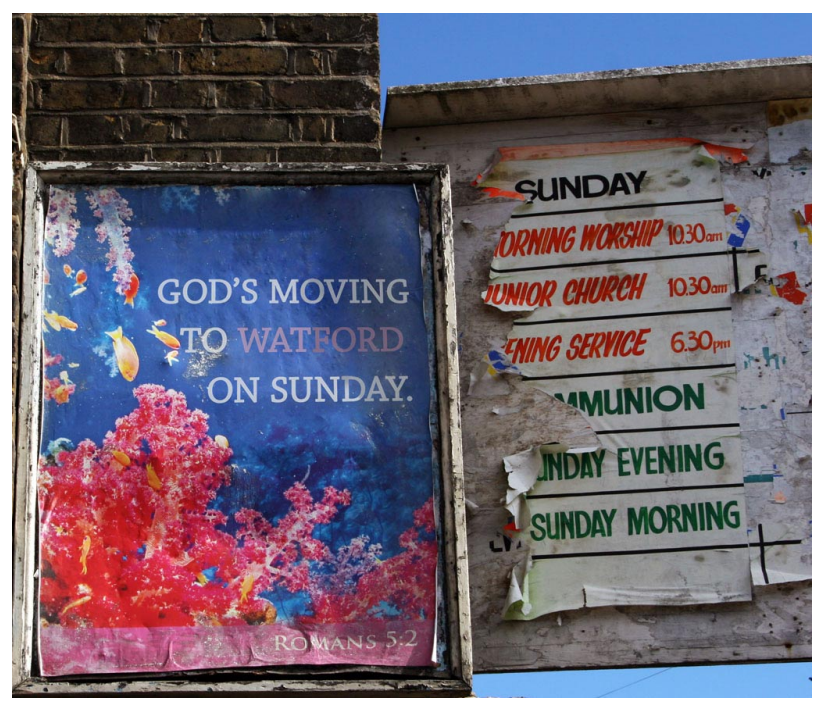

(a)

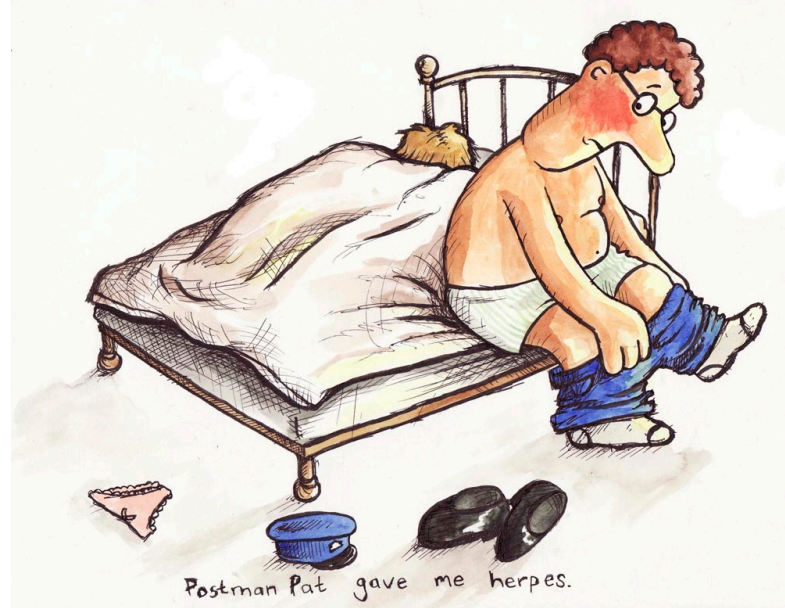

(b)

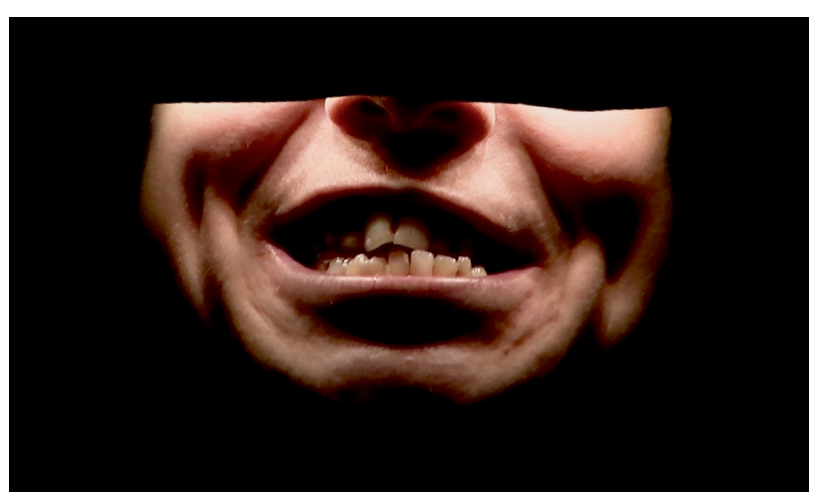

(c)

Figure 1: a) 'God's Moving to Watford on Sunday' by Jess Thom, b) 'Postman Pat Gave Me Herpes' by Luke Turner, c) Photograph from a performance of Samuel Beckett's 'Not I' by Touretteshero at Battersea Arts Centre. Courtesy of: James Lyndsay would benefit from being considered entirely distinct [8]. Other researchers describe a tension between musicality and utility, where sonifications become decreasingly useful and transparent as they become increasingly musical and satisfying $[9,10]$, although Vickers has recently brought this dualism into question [11]. A consensus does form, however, around the use of aesthetics to enhance the communicative and expressive qualities of an auditory display and to reduce fatigue $[12,13]$. This observation has led to numerous calls for interdisciplinary collaboration to encourage the integration of artistry and craft into sonification research $[14,15,16]$.

Sonification and music can be considered on a continuum between representation and abstraction, or infomatica and musica as described by Vickers and Hogg [7]. Representation tends to emphasise an information theoretic approach, viewing the auditory system as a communications channel and prioritising the faithful expression of information with little regard for aesthetics. Conversely, abstraction is concerned with aesthetics over representation, as the underlying information may be symbolic or serve only as inspiration. The extremes are easily identified, for example, representation is a priority for sensory substitution devices that translate image into sound (e.g. 'The vOICe' [17]), and abstraction is emphasised in data inspired music (e.g. Alvin Lucier's 'Music for Solo Performer' [18]) where sonification manifests as an artistic device. Common to all sonifications is the intention of the designer/artist to convey information with sound, combined with the delegation of some aspect of the aural fabric to a data source [19]. If the mapping is intended to have scientific utility, the data points of interest must be rendered faithfully such that they may be inferred by listeners.

The aim of this work is to explore a mid-point on the continuum from representation to abstraction, prioritising sonification aesthetics without compromising the accuracy and legibility of the underlying data with an artificial 'musical' framework. We believe this approach has the potential to increase the agency of sonification in terms of access, usability and ergonomics through careful design choices rather than exclusively artistic activity. With this in mind, inspiration is taken from the field of sound design, with elements from the (somewhat related) field of acousmatic music. An outline of this approach is laid out in Section 4.2 below.

The remainder of this paper describes the creation of a data inspired composition that translates a year of Jess's ticcing fits into a sound piece. Many aspects of the data within the records are sonified to preserve and convey the frequency and relentlessness of these episodes, with aesthetic and sound design choices that help to bring this data into the human realm, inviting listeners to contemplate the lived experience of Tourette's.

\section{THE ALCHEMY OF CHAOS}

The Alchemy of Chaos was prompted when Jess Thom (Touretteshero) was invited to deliver a keynote at the Royal Albert Hall in the UK at the TEDxAlbertopolis event. It had been a year since Jess had started to experience intensive ticcing fits and she wanted to share this aspect of her condition at the event.

At the onset of these episodes, Jess began keeping detailed records to look for trends, patterns and possible causes with her medical consultants. During a ticcing episode, any part of Jess's body may move, shake, contort or lock into painful positions, which can resemble an epileptic seizure but with major differences: Jess remains fully conscious and aware of her surroundings and although unable to speak she is normally able to communicate 


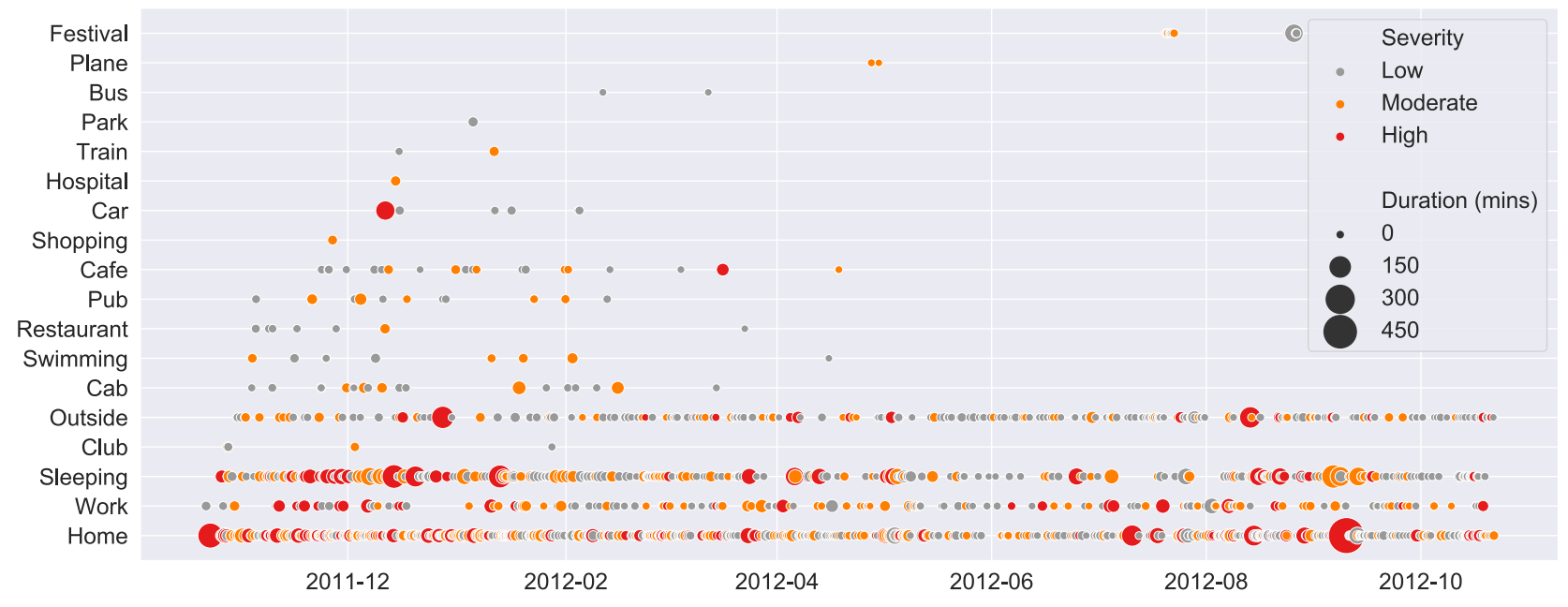

Figure 2: Visualisation of the ticcing fit data showing the location, intensity, duration and time of each episode

by blinking. Ticcing fits can occur at any time and in any location, they vary in severity, duration and frequency and are likely to result in injury without the help of a support worker. While these episodes can be physically exhausting, as soon as they calm, Jess is immediately able to resume the activity she was attending to previously.

Unlike Jess's vocal tics that inspire the majority of the Touretteshero artwork, humour is notably absent from this aspect of her symptoms. However, the desire to create an artform from the records was shared with the co-authors of this paper and subsequent discussions resulted in plans for a data inspired sound piece. The aim was to translate the alphanumeric information of the records into music that preserves, humanises and coveys this aspect of Jess's everyday lived experience.

\subsection{Data}

Following each episode, Jess or her support worker would note the start date, time, duration, severity (low, moderate or high) and location along with some additional notes (i.e. body parts affected and whether speech was lost). Records were transcribed and shared with the project team in spreadsheet format. The complete data set contained records for 2011 episodes that took place between 21st October 2011 and the same day one year later.

The data was initially examined, converted to CSV (comma separated values) and preprocessed to identify and correct any transcription errors and inconsistencies that were found. For example, typos, wording and capitalisation variations, and ordering errors (owing to mixed 24/12 hour clock entries). Occasionally, entries were recorded with two locations because the episode may have started in one location and ended in another, e.g. 'Outside/Cab'. Alternatively, these items were a result of the emergent nomenclature mixed with more specific labels, e.g., 'Outside/Park'. Instances of the former were replaced with the start location of the episode and instances of the latter were replaced with the more specific location. A plot visualising the data is provided in Figure 2.

\subsection{Sound Design}

Several suggestions and guidelines that define sonification have been proposed previously, for example, Hermann's definitions [20] set out criteria that promote objectivity and reproducibility. Other authors have provided less prescriptive definitions that highlight the importance of authenticity and the preservation of relative time structures [10].

In this work, sound design took a compositional approach, drawing inspiration and working practices from acousmatic music, acoustic ecology and sound design. Precise timing was considered a priority, with the aim of preserving and representing the relative timing of events within the piece. Consequently, playback time for the piece was considered in units of days per minute $(\mathrm{dpm})$ with the sound events for each episode scheduled in relation to its timestamp within the data.

The general approach for generating the sound content took a Foley approach, using primarily short, clearly identifiable, closemiked sounds to construct the dominant audio texture. For each location, a representative symbolic sound (or auditory icon [21]) was selected. Particular inspiration was taken from the pioneering sound design work of Walter Murch on several seminal films made in the 1970s by directors such as Francis Ford Coppola and George Lucas [22]. The soundtracks to these films blur the boundaries between the traditionally-demarked areas of sound design and music, with one often taking on the role of the other. For example, in the opening sequence of Lucas's TXH1138, a sequence of controls on a console light up and the corresponding 'beep' tones form a melodic contour that functions as part of the music, offering a segue into the following scene where the same tones become the bell of an elevator. In the 'Tiger Scene' in Coppola's Apocalypse Now, high-pitched insect sounds fulfil the tension-building function more commonly facilitated with high 'Psycho' inspired strings.

In 'The Alchemy of Chaos' recognisable sounds are used to represent features of the data being sonified. Harmonic principles were employed in selecting these sounds, and in some cases manipulation (re-tuning), such that they collectively form harmonic, melodic patterns. This is a good example of sound design fulfill- 
ing the 'function' of music - beyond this choice, no other musical logic was imposed upon the data, but this design choice enables the result to still sound 'musical'. Note that the pitch of the sounds has no significance to the sonification, allowing this creative 'liberty' to be taken without compromising the transparency of the sonification. Where parameters do have data significance, no such interventions were made.

Another of Murch's sound design principles is also adopted here, defined by Chion as elements of auditory setting (EAS), that is:

'a punctual source... which help to define a film's space by means of specific, distinct small touches. Typical sounds of the auditory setting are the faraway barking of a dog, or the ringing of a phone in the office next door, or a police car siren' - [23], p54

EAS allows locations to be defined with minimal sonic references, an efficient approach allowing much flexibility and space for other elements of the sound design/music. In this sonification, EAS are used to define the location of each ticcing fit, whilst allowing other sound parameterisations, timing, amplitude and reverberation to define the start time, intensity and duration of each episode respectively.

These short sounds, arranged into distinct categories according to the location of the tics, resemble Pierre Schaeffer's l'objets sonores. Our intention here is somewhat at odds with Schaeffer's (a founding principle of musique concrete). His idea, rooted in the phenomenology of Husserl, was that sounds might be divorced from their source and any a priori meaning, and treated as abstract plastic entities. In this sonification, the sounds are explicitly intended to have 'meaning' (as representing each location). This corresponds more with 'causal listening' as defined by Shaeffer rather than 'reduced listening'. However, the parameterisation of timing, amplitude and reverb is informed by Schaeffer's approach, as subsequently expanded by Denis Smalley in his writings on Spectromorphologys [24]. Certainly, the approach to sonification taken here has more in common with musique concrete than it does with more traditional forms of music.

For each location in the data set, a symbolic sound was chosen. In most cases, these were recorded by Jess in the locations where many of these episodes may have taken place, establishing a link to the acoustic ecology of Jess's day-to-day environment [25]. In some instances this was impractical ('club') or inappropriate ('hospital'), in which case, suitable substitutions were found. A complete list of locations, their incidence and the representative sounds is provided in Table 1 .

\subsection{System Overview}

The sound piece was made possible with the development of two applications, a Data Player and a Sampler. Both applications ran on a single machine and communicated by Open Sound Control (OSC) [26] over a network datagram socket. The system architecture is shown in Figure 3.

The Data Player was a simple application developed in $\mathrm{C}++$ using the Juce library [27] that opened, parsed and 'played' the data in a variety of modes. Accepting as input a CSV file containing the episode data, the application produced as output UDP packets containing an OSC message for each episode with the address pattern /episode and the arguments shown in Table 2.

The data playback rate was in units of $d p m$ as described in section 4.2. For the final audio piece, this was set to $60 \mathrm{dpm}$ where

\begin{tabular}{||lcl||}
\hline Location & Incidence & Sound \\
\hline \hline Bus & 2 & bus - interior and exterior \\
Cab & 20 & cab - exterior and exterior \\
Café & 21 & cups and saucers \\
Car & 8 & ignition and engine \\
Club & 7 & indicative music clip \\
Festival & 37 & crowd \\
Home & 967 & 'soundmarks' from Jess's home \\
Hospital & 1 & hospital machine (from library) \\
Outside & 304 & birdsong \\
Park & 1 & children playing \\
Plane & 2 & plane \\
Pub & 16 & crowd - interior \\
Restaurant & 7 & knives and forks \\
Shopping & 1 & musak clip \\
Sleeping & 398 & Jess's alarm clock \\
Swimming & 10 & swimming pool \\
Train & 2 & station announcement \\
Work & 207 & typing \\
\hline
\end{tabular}

Table 1: List of episode locations and their sound representations

\begin{tabular}{||lll||}
\hline Argument & Type & Description \\
\hline \hline counter & int32 & index of episode in the dataset $(0-2010)$ \\
duration & int32 & rounded to nearest minute \\
severity & int32 & where $0=$ low, $1=$ moderate, $2=$ high \\
location & int32 & $0-17$ see Figure 2
\end{tabular}

Table 2: OSC message format for each episode

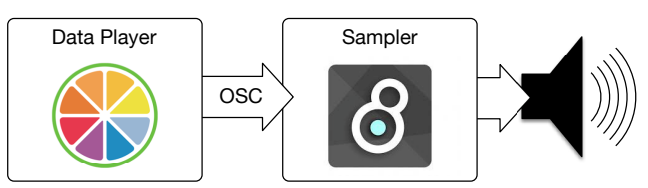

Figure 3: System block diagram showing Data Player and Sampler applications

each day can be imagined as a beat at a tempo of $60 \mathrm{bpm}$ and the resulting year of ticcing episodes translates to 6:05 seconds. To ensure that messages were precisely timed, all event messages were scheduled and sent on a high priority timing thread.

The application GUI provided controls for loading a data file, toggling playback positioning/resetting the playhead and playback rate. Three timing modes were also available and explored: Proportional, where the timing structure and intervals are maintained precisely, as described above; Day Beat, where all events in a single day are played simultaneously on the onset of their respective day beat; and Day Beat Quantize, where events are quantized to the nearest semiquaver of their respective day beat. After some initial experimentation only the Proportional method was used as relative timing was considered more important than the aesthetic rhythmic qualities that the other methods introduced.

The Sampler was a simple audio file playback system created in Max/MSP that received and decoded the OSC messages sent from the Data Player and triggered audio files in response. The sampler was loaded with the Foley samples representing each location (so, for example 'café' might be represented by the clink of a cup on a saucer). Rather than use a single sample for each 


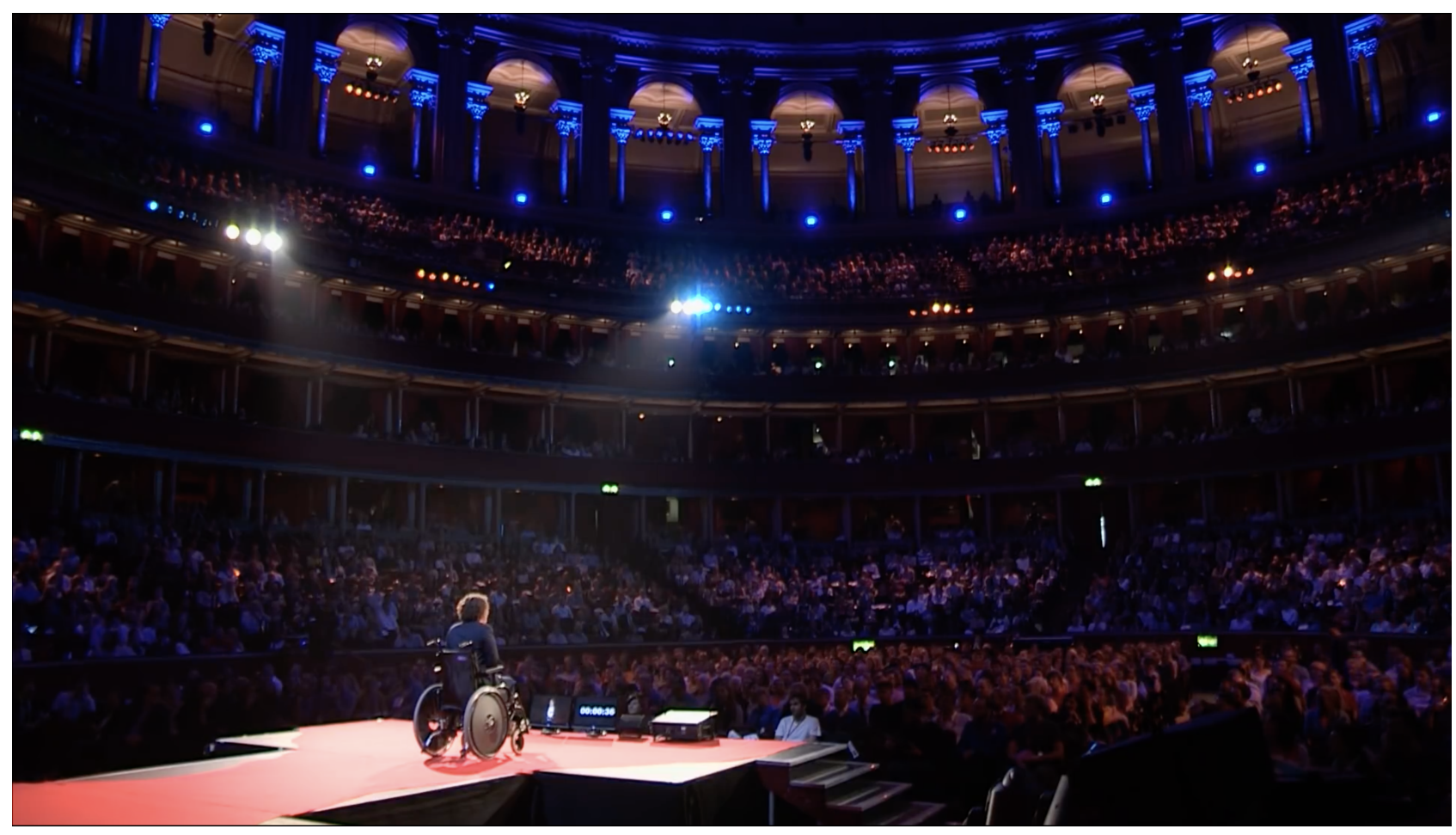

Figure 4: Still taken from the video 'Tourette's syndrome - why it doesnt define me - TEDxAlbertopolis', showing Touretteshero addressing the audience at the Royal Albert Hall

location, a small library of Foley sounds (between 4 and 20) were loaded into a sound bank and could be loaded and triggered in a randomised order, a technique used in game audio to avoid fatiguing and artificial repetition.

The sample amplitude is then scaled to reflect the intensity of the episode before being summed into a simple reverberator with the diffusion time scaled to reflect the duration of each episode (which was deemed aesthetically preferable to actually lengthening the sounds using pitch shift or timestretch).

\section{QUALITATIVE REFLECTION}

An excerpt of the resulting sonification ${ }^{1}$ was played to an audience of 5000 delegates at Royal Albert Hall as part of the TEDxAlbertopolis ${ }^{2}$ event, see Figure 4.

Listening to the piece, it is possible to identify the rhythm of passing days from the alarm clock sound, signifying the 'Sleeping' location. With episodes occurring every four hours, on average, most days included an entry taken at night and this regular pulse persists throughout the sound piece, contextualising the time structure and conveying the frequency and disruptive nature of these episodes.

As commented by Jess in her writings [28], seasonal changes are also recognisable as the piece progresses, with the number of 'Outside' sounds increasing in frequency from the midway mark

\footnotetext{
${ }^{1}$ Which can be accessed here:

https://soundcloud.com/josephhyde/touretteshero-whole-year

${ }^{2}$ Which is available here:

https://www.youtube.com/watch?v=_jmTlQld2Z8
}

as dates pass into spring and summer. The piece is noticeably calmer and sparse during this period, as can be observed in Figure 2 where, in general, episodes tended to occur less frequently, were less severe and shorter in duration. An observation again confirmed by Jess, who notes that ticcing fits can intensify at times of stress and anxiety, symptoms that are both relieved by the longer days and warmth of the summer season.

The sound palette can also be heard evolving throughout the piece with the full range of locations and chaotic sound textures present in the first half, settling into a consistent sound texture from the mid-point onward. This evolution in sound ecology reflects Jess adaption as these ticcing fits became a permanent and uncontrollable aspect of everyday life. As episodes can occur at any time and in any location, Jess's behaviour change is signified by a stabilising sound palette as certain locations within the data set (e.g. swimming, eating out, clubbing, etc.) disappear. In her writings about the sound piece, Jess comments on this change and notes that the ticcing fits have led to a reduced sense of independence and freedom [28]. While the tics themselves cannot be controlled, it is possible to control the factors that make them more manageable or less likely to occur.

\section{CONCLUSION}

This paper documents a translation of a year of intensive ticcing episodes (or 'ticcing fits') into a 6:05 minute sound art piece that was played to an audience of 5000 people at the Royal Albert Hall in the UK. The data included the time, location, duration and intensity of 2011 ticcing fits and were recorded by Jess Thom, who 
spreads awareness of Tourette's and its challenges by sharing the challenges and creativity of her tics through the Touretteshero organisation. The aim of this work was to convert a data set representing a great deal of discomfort, into something creative and engaging, while preserving and conveying the relentlessness and lived experience of this aspect of Jess's condition. The collaborative team used sonification techniques to represent the recorded data variables as sound, drawing inspiration and techniques from sound design for film to enhance the aesthetics of the piece without compromising the data representation. In particular, Chion's elements of auditory setting are used to rapidly convey a sense of location for each ticcing fit through short, punctual sound icons. The paper also introduces the process of grouping sonification parameters into those that have elements of 'data significance' and those that do not, with aesthetic and creative interventions limited to sound parameters that do not impact on the data transparency of the sonification.

\section{ACKNOWLEDGMENT}

TJM and JH acknowledge support from Leverhulme Trust, Royal Society, the British Academy and the Royal Academy of Engineering under grant APX $\backslash \mathrm{R} 1 \backslash 180118$. The authors would like to thank Dr David Glowacki, Alex Jones and the members of the Glowacki Group and CT Lab.

\section{REFERENCES}

[1] R. H. Bitsko, J. R. Holbrook, S. N. Visser, J. W. Mink, S. H. Zinner, R. M. Ghandour, and S. J. Blumberg, "A national profile of tourette syndrome, 2011-2012," Journal of developmental and behavioral pediatrics, vol. 35 , no. 5, pp. 317 22, 2014.

[2] J. S. Stern, "Tourette's syndrome and its borderland," Practical neurology, vol. 18, no. 4, pp. 262-270, 2018.

[3] H. Smith, J. Fox, and P. Bunton, "The lived experiences of individuals with tourette syndrome or tic disorders: A metasynthesis of qualitative studies," British Journal of Psychology, vol. 106, no. 4, 2014.

[4] M. M. Robertson, "Tourette syndrome," Psychiatry, vol. 4, no. 8, 2005.

[5] J. Thom, Welcome to Biscuit Land: A Year in the Life of Touretteshero. Souvenir Press, 2012.

[6] P. Vickers, "Ars informatica - ars electronica: Improving sonification aesthetics," in Understanding and Designing for Aesthetic Experience (workshop at The 19th British HCI Group Annual Conference), 2005.

[7] P. Vickers and B. Hogg, "Sonification abstraite / sonification concrète: An 'aesthetic perspective space' for classifying auditory displays in the ars musica domain," in Proceedings of the 12th International Conference on Auditory Display, 2006.

[8] C. Scaletti, "Sonification $\neq$ music," in The Oxford Handbook of Algorithmic Music. Oxford Univeristy Press, 2018.

[9] T. Bovermann, J. Rohruber, and A. de Campo, "Laboratory methods for experimental sonification," in The Sonification Handbook. Logos Verlag, 2011.
[10] S. Gresham-Lancaster, "Relationships of sonification to music and sound art," AI and Society, vol. 27, no. 2, 2012.

[11] P. Vickers, "Sonification and music, music and sonification," in The Routledge Companion to Sounding Art. Routledge, 2016.

[12] G. Kramer, "An introduction to auditory display," in Auditory Display: Sonification, Audification and Auditory Interfaces. Addison-Wesley, 1994.

[13] P. Vickers, "Lemma 4: Haptic input + auditory display = musical instrument?" in Proceedings of the First International Conference on Haptic and Audio Interaction Design, 2006.

[14] S. Barrass and G. Kramer, "Using sonification," Multimedia Systems, vol. 7, no. 1, 1999.

[15] M. Barra, T. Cillo, A. De Santis, U. F. Petrillo, A. Negro, and V. Scarano, "Multimodal monitoring of web servers," IEEE MultiMedia, vol. 9, no. 3, 2002.

[16] S. Barrass, "Sonifications for concert and live performance," AI and Society, vol. 27, no. 2, 2012.

[17] P. B. L. Meijer, "An experimental system for auditory image representations," IEEE Transactions on Biomedical Engineering, vol. 39, no. 2, pp. 112-121, 1992.

[18] V. Straebel and W. Thoben, "Alvin lucier's music for solo performer: Experimental music beyond sonification," $\mathrm{Or}$ ganised Sound, vol. 19, no. 1, pp. 17-29, 2014.

[19] P. Sinclair, "Sonification: what where how why artistic practice relating sonification to environments," AI and Society, vol. 27, no. 2, 2012.

[20] T. Hermann, "Taxonomy and definitions for sonification and auditory display," in Proceedings of the 14th International Conference on Auditory Display, 2008.

[21] W. W. Gaver, "Auditory icons: Using sound in computer interfaces," Human-Computer Interaction, vol. 2, no. 2, 1986.

[22] M. Ondaatje and W. Murch, The conversations: Walter Murch and the art of editing film. A\&C Black, 2002.

[23] M. Chion, Audio-Vision: Sound on Screen. Columbia University Press, 1994.

[24] D. Smalley, "Spectromorphology: Explaining soundshapes," Organised sound, vol. 2, no. 2, 1997.

[25] A. Polli, "Soundscape, sonification, and sound activism," $A I$ and Society, vol. 27, no. 2, 2012.

[26] M. Wright and A. Freed, "Open sound control: A new protocol for communicating with sound synthesizers," in Proceedings of the International Computer Music Conference, 1997.

[27] R. Ltd. The juce library. [Online]. Available: https://juce.com

[28] J. Thom. Rah. [Online]. Available: https://www. touretteshero.com/2013/09/23/rah/ 